\title{
NEUROENDOCRINE CARCINOMA OF THE NASAL CAVITY - A CASE REPORT AND BRIEF REVIEW OF LITERATURE.
}

Madhura D. Paricharak, Sunita B. Patil, P. V. Purohit, Shilpa Narchal, S. S. More

1. Post Graduate Student, Department of Pathology, D. Y. Patil Medical College. Kolhapur.

2. Assistant Professor, Department of Pathology, D. Y. Patil Medical College. Kolhapur.

3. Consultant Pathologist \& Microbiologist, Kolhapur.

4. Post Graduate Student, Department of Pathology, D. Y. Patil Medical College. Kolhapur.

5. Professor \& Head, Department of Pathology, D. Y. Patil Medical College. Kolhapur.

\section{CORRESPONDING AUTHOR}

Dr. Madhura D. Paricharak

Post Graduate Student, Department of Pathology,

D. Y. Patil medical college, Kasaba Bawada,

Kolhapur - 416006, Maharashtra

E-mail: madhupari02@yahoo.co.in

Ph: 00917798881426.

ABSTRACT: Neuroendocrine carcinoma of the head and neck are rare aggressive tumors and pose a diagnostic challenge. Differentiation from nasopharyngeal malignancies is difficult due to similar morphological characteristics and small biopsy specimen. Distinguishing is important because of difference in natural history, prognosis and treatment among these neoplasms. We report a 60 year old man with small cell neuroendocrine carcinoma of nasal cavity. Histology reveals highly cellular tumor with small nuclei with stippled chromatin pattern and scanty cytoplasm. Immuno-histochemical stain showed positivity for chromogranin A and cytokeratin, confirmed our diagnosis.

KEY-WORDS: Neuroendocrine tumor, small cell carcinoma, nasopharyngeal malignancy.

KEY MESSAGE: Sinonasal neuroendocrine carcinoma has overlapping histomorphological features of other tumors like olfactory neuroblastoma, lymphoma and undifferentiated carcinoma. Ancillary techniques like immunohistochemical studies help in confirming the diagnosis and subcatagorising neuroendocrine carcinomas.

INTRODUCTION: Small cell neuroendocrine carcinoma is a rare malignancy of the head and neck. It represents $0.5-1 \%$ of epithelial cancers of head and neck region. The 2005 World Health Organisation classification of Head and Neck Tumors categorizes these neoplasms as typical carcinoid tumor, atypical carcinoid tumor, small cell carcinoma neuroendocrine type (SNEC) and neuroendocrine carcinoma "not otherwise specified".1,2,3 Patients with neuroendocrine malignancies usually present with locally advanced disease. These neoplasms can not generally be distinguished based on clinical or radiological criteria and hence require histopathological evaluation and further immunohistochemical confirmation of neuroendocrine differentiation. It should be differentiated from other sinonasal tumors like esthesioneuroblastoma (ENB), sinonasal undifferentiated carcinoma (SNUC) and lymphomas. 4,5

CASE HISTORY: A $60 \mathrm{yr}$ old male came with complaints of epistaxis and headache of 1 month duration. The patient was non-smoker and had not been exposed to radiation or environmental irritants. Patients medical history was not contributory. 
On clinical examination, fleshy, friable, highly vascular mass in the right nasal cavity. There were no obvious, palpable cervical lymph nodes and no distant metastasis was found. The mass was subjected for incisional biopsy and specimen was sent for histopathological evaluation.

Computer tomography revealed homogenous tumor in the nasal cavity extending towards orbit and base of skull.

On gross examination, specimen consisted of few soft pale brown tissue bits weighing about 1gm. Histologic examination revealed highly cellular tumor exhibiting solid, nesting, trabecular pattern. No rosette formation seen. Nuclei were small, round, monomorphic with stippled chromatin pattern. Cytoplasm was scanty, eosinophilic. Mitotic figures were frequent. Areas of necrosis were observed. There was no definite lymphovascular or perineural permeation.

We considered paraganglioma, neuroendocrine tumors and ENB as differential diagnosis. We could arrive on definitive diagnosis by doing immunochemical studies. The tumor turned out to be positive for Cytokeratin and Chromogranin A and negative for Epithelial membrane antigen (EMA).

DISCUSSION: Primary neuroendocrine carcinoma of the nasal cavity is rare and represents a histological spectrum of differentiation. It appears in the $6^{\text {th }} 7^{\text {th }}$ decades of life; unusual below 40 yrs of age. ${ }^{4-6}$ In a study by Silva et al, the mean age of patients of SNEC was 50 years and that for ENB was 20 years. $^{7}$ The most common site for this tumor in the head and neck region is the larynx. ${ }^{4,5}$ SNEC is defined as high grade epithelial neoplasm with neuroendocrine differentiation, at both immunohistochemical and ultrastructural levels. ${ }^{4}$

Neuroendocrine carcinoma of the head and neck may be stratified into a number of subsets on the basis of appearance under light microscopy, immunohistochemical staining, ultra structural findings and clinical course. These tumors are having an epithelial or neural origin. The neural subgroup includes paraganglioma and epithelial subgroup includes typical carcinoid, atypical carcinoid and small cell neuroendocrine carcinoma. Cytokeratin is the marker to differentiate between neural and epithelial neuroendocrine tumors. ${ }^{4}$ There is wide range at presention and most common symptoms are epistaxis and headache, as observed in our case. Other common symptoms are hoarseness, dysphagia, paresthesia, anosmia, facial pain, proptosis, palpable facial mass and exophthalmos.5,6 Most tumors arise in the nasal cavity but may extend into adjacent sinuses. Local extension is seen in skull, orbit and brain whereas distant metastasis are seen in cervical lymph nodes, lungs, liver and bone. ${ }^{4,5}$ Approximately $50 \%$ of patients have cervical metastasis on initial presentation. ${ }^{4}$ Small cell carcinoma of the head and neck is also notable for hematogenous dissemination. Exceptionally the tumor may be associated with a paraneoplastic syndromes like Cushing, Eaton-Lambert and Schwartz-Bartter syndromes. ${ }^{4,6}$

Neuroendocrine tumors are usually unencapsulated covered by an uninvolved, although frequently ulcerated mucosal surface. 5 Different histological patterns are seen including organoid, trabecular, cords, sheets, ribbons, pseudoglands and rosette formation, solid and single file pattern. Lymphovascular, perineural and soft tissue invasion is common. Nuclei are small, uniform with stippled chromatin pattern and little or no cytoplasm. Frequent mitosis, necrosis and apoptosis are common.5,6 Kameya et al, described four sinonasal neuroendocrine carcinomas among them three were reported as malignant carcinoid and one as small cell carcinoma. The authors provided no specific mitotic count other than "rare" and "numerous". 
Similarly in our case mitosis were frequent. Hyams et al, proposed importance of distinguishing SNEC from ENB as their relationship remains confusing and controversial. SNEC lacks lobular architecture, fibrovascular septa, neurofibrillary stroma and does not contain neural or olfactory rosettes. The anaplastic cells of SNEC have negligible cytoplasm, high nucleo/cytoplasmic ratio, round or oval dense hyperchromatic nuclei, numerous mitotic figures and apoptotic cells accompanied by extensive areas of necrosis. ${ }^{1}$ Ultrastructurally SNEC is characterized by membrane bound, electron -dense neurosecretory granules ranging from 50$200 \mathrm{~nm}$. ENB shows presence of dense core neurosecretory granules measuring $50-250 \mathrm{~nm}$ in diameter and neurite-like cell processes containing neurofilaments and neurotubules. SNUC have occasional small desmosomes and rare dense core granules. ${ }^{6}$

Conventional microscopy is generally insufficient for the diagnosis of neuroendocrine carcinoma, as it shares histological features with other neoplasms, such as lymphomas, SNUC, ENB and primitive neuro ectodermal tumors (PNET). ${ }^{5,9}$ Immunohistochemistry aids in distinguishing between small cell tumors of different histogenetic origins. Markers such as chromogranin, synaptophysin and neuron specific enolase are frequently expressed by neuroendocrine tumors. ENB is excluded by negative staining for S-100 protein and Neuron specific enolase (NSE). Malignant lymphoma by negative CD56 \& leukocyte common antigen. SNUC is positive for cytokeratin and variable positivity for NSE. PNET can be ruled out by negative CD 99 and vimentin.4,5,9

Menon et al, reported 37 cases, of which 14 cases of SNUC, 14 ENB and 9 cases of SNEC. The cases of SNUC were immunopositive for cytokeratin, epithelial membrane antigen and weakly for neuron specific enolase. SNEC showed strong reactivity with epithelial and neuroendocrine markers whereas ENB demonstrated immunoreactivity to synaptophysin and chromogranin strongly, with weak to negative expression of epithelial markers. ${ }^{10}$

Small cell neuroendocrine carcinomas are aggressive tumours with a poor prognosis and frequent local recurrence and distant metastasis despite multimodal therapy. Nasopharyngeal carcinoma is to some extent preventable. It shows a very strong association with Epstein Barr virus infection. Nasopharyngeal carcinoma is generally responsive to radiation therapy and the clinical outcome has greatly improved over the years, due to refinements in staging and to improved therapy protocols. ${ }^{6}$

CONCLUSION: Small cell carcinoma neuroendocrine type - sinonasal has overlapping histomorphological features of other tumors like olfactory neuroblastoma, lymphoma, and undifferentiated carcinoma. Ancillary techniques like immunohistochemical studies help in confirming the diagnosis and subcatagorising neuroendocrine carcinomas.

ACKNOWLEDGEMENT: We sincerely thank Dr. Jaydeep Pole, Oncopathology center Miraj, for technical support and assistance.

\section{REFERENCES:}

1. Weinreb I, Perez-Ordonez B. Non-small cell neuroendocrine carcinoma of the Sinonasal Tractand Nasopharynx. Report of 2 cases and review of the literature. Head Neck Pathol.2007 September;1(1):21-26. 
2. Likhacheva A, Rosenthal DI, Hanna E, Kupferman M, DeMonte F, El-Naggar AK. Sinonasal neuroendocrine carcinoma: impact of differentiation status on response and outcome. Head and Neck Oncology 2011;3:32-37.

3. Iacovou E, Chrysovergis A, Eleftheriadou A, Yiotakis I, Kandiloros D. Neuroendocrine carcinoma arising from the septum. ACTA Otorhinolaryngologica Italica 2011;31:50-53.

4. Subha S. Neuroendocrine carcinoma of the Nasopharynx-A case report. Medical and Health Science Journal. Vol 9;2011:101-103.

5. Lin TC,Tsai MH. Poorly differentiated neuroendocrine carcinoma of the nasal cavity. Mid Taiwan J Med.2009;14:41-45

6. Barnes L, Eveson JW, Reichart p, Sidransky D (eds). Pathology and genetics of Head and Neck Tumors. World Health Organisation Classification of tumors. Lyon,France:IARC Press,2003.

7. Silva EG, Butler JJ, Mackay B, Goepfert H. Neuroblastomas and neuroendocrine carcinomas of the nasal cavity: A proposed new classification. Cancer 1982;50:23882405.

8. Kameya T, Shimosato Y, Adachi I, Abe K, Ebihara S, Ono I. Neuroendocrine Carcinoma of the paranasal sinus. A morphological and endocrinological study. Cancer. 1980;45:3309.

9. Wenig BM.Tumors of the upper respiratory tract. In:Fletcher CDM.ed. Diagnostic histopathology of tumors.3rd ed. Philadelphia,USA:Churchill Livingstone Elsevier;2007:83-180.

10. Menon S, Pai P, Sengar M, Aggarwal JP, Kane SV. Sinonasal malignancies with neuroendocrine differentiation: Case series and review of literature. Indian J of Pathol Microbiol 2010 Jan-Mar;53(1):28-34.

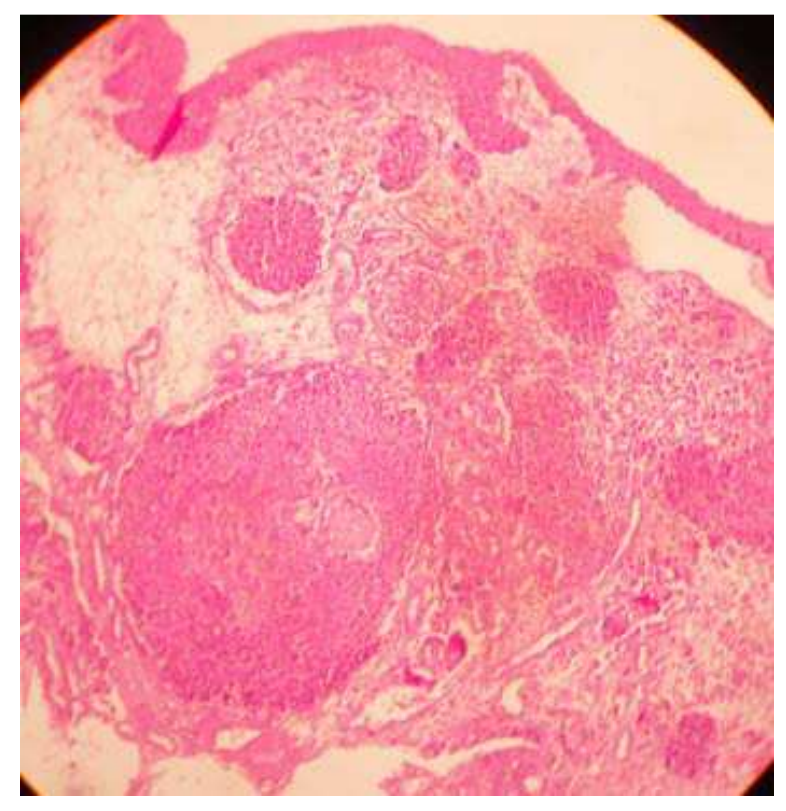

Photomicrograph showing small round tumor cells arranged in nests with overlying intact nasal mucosa. 


\section{CASE REPORT}

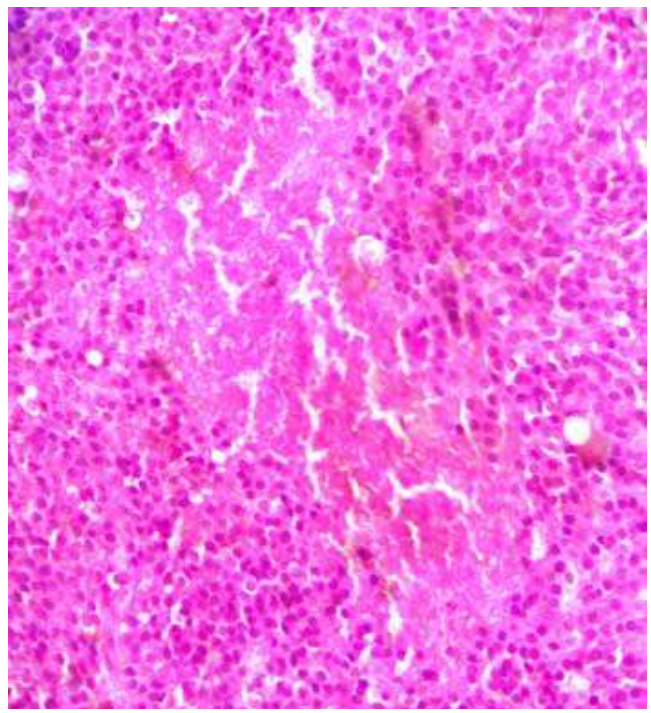

Photomicrograph showing monomorphic tumor cells with dark nuclei and foci of necrosis

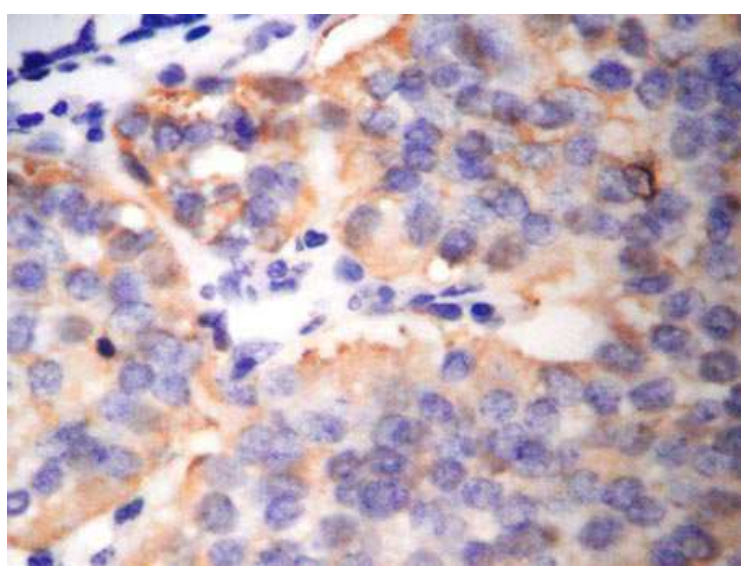

Photomicrograph showing positive staining for chromogranin-40X

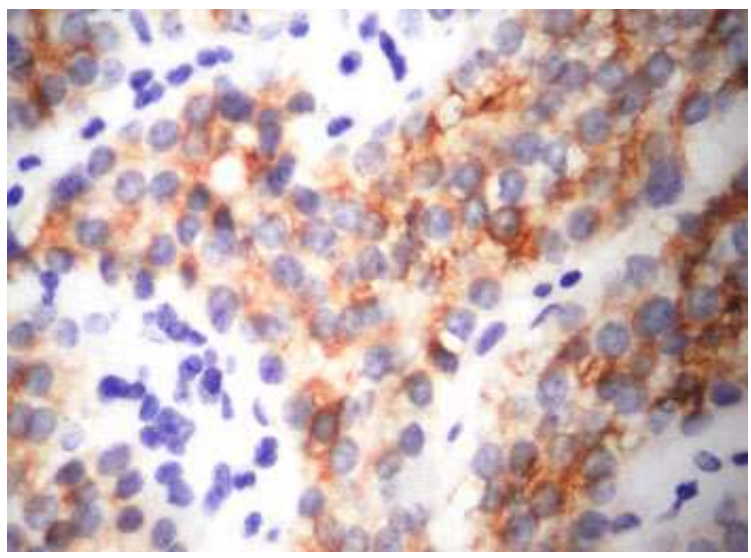

Photomichrograph showing positive stainin for cytokeratin-40X 